\title{
Contourner le vide: écriture et judéité(s) après la Shoah, dir. F. DAINESE et E. QUAGLIA
}

\section{Stefano Genetti}

\section{OpenEdition}

\section{Journals}

\section{Edizione digitale}

URL: https://journals.openedition.org/studifrancesi/45463

DOI: 10.4000/studifrancesi.45463

ISSN: 2421-5856

\section{Editore}

Rosenberg \& Sellier

\section{Edizione cartacea}

Data di pubblicazione: 1 août 2021

Paginazione: 401-402

ISSN: 0039-2944

\section{Notizia bibliografica digitale}

Stefano Genetti, «Contourner le vide: écriture et judéité(s) après la Shoah, dir. F. DaINESE et E. QuagliA», Studi Francesi [Online], 194 (LXV | II) | 2021, online dal 01 septembre 2021, consultato il 15 octobre 2022. URL: http://journals.openedition.org/studifrancesi/45463 ; DOI: https://doi.org/10.4000/ studifrancesi. 45463

Questo documento è stato generato automaticamente il 15 octobre 2022

\section{(c) $($ ) $(9)$}

Creative Commons - Attribuzione - Non commerciale - Non opere derivate 4.0 Internazionale - CC BY NC-ND 4.0

https://creativecommons.org/licenses/by-nc-nd/4.0/ 


\title{
Contourner le vide: écriture et judéité(s) après la Shoah, dir. F. DAINESE et E. QUAGLIA
}

\author{
Stefano Genetti
}

\section{NOTIZIA}

Contourner le vide: écriture et judéité(s) après la Shoah, dir. F. DAINESE et E. QUAGLIA, Firenze, Giuntina, 2020, $123 \mathrm{pp}$.

1 Il volume raccoglie gli Atti della giornata di studio Judéités sans Judaïsme après la Catastrophe (Verona, 2 febbraio 2017) connessa al pluriennale seminario "L'Alphabet de la Shoah". Memoria e narrazione, organizzato da Rosanna Gorris e dalle curatrici. Pourquoi la judéité?, si chiedono queste ultime nella documentata Introduction (pp. 7-14; si veda la Bibliographie essentielle che precede l'Indice dei nomi), riprendendo il termine introdotto da Albert Memmi per distinguere il judaïsme in quanto dottrina dalla judéité in quanto tratto identitario svincolato dal senso di appartenenza a una fede o a una comunità. In conseguenza del vuoto lasciato dallo sterminio, la judéité si inscrive sotto il segno del patrimonio violato e negato, quasi «un résidu de transmission», scrivono F. Dainese e E. Quaglia, «qui se dépose, après décantation, au fond liquide de l'identité» (p. 8). Alla ricomposizione di un'identità dispersa concorrono le strategie, dense di implicazioni sia esistenziali che estetiche, messe in atto dalla scrittura letteraria. La riflessione sul linguaggio e sulle modalità espressive costituisce infatti un filo conduttore di questa raccolta: dall'omologia tra mondo e libro su cui si basa l'identità ebraica, al confluire di letteralità e letterarietà («L'Être à la lettre», p. 20), al rapporto con la «judéo-langue» («Comme Perec bégaie son hébreu en inventant une lettre hébraïque qui n'esiste pas, Doubrovsy balbutie son yiddish», p. 45); dalla centralità della parola juif, del testo biblico e della riflessione sul gesto di scrivere come mediazione per accedere alla 
memoria individuale e collettiva (p. 82, p. 93) alla messa in discussione dell'autofinzione come risorsa per colmare le lacune di un racconto inenarrabile (p. 97).

2 Se, quasi a sottolineare la vocazione comparatistica degli studi sul tema, il volume si chiude con il saggio di Alberto CAVAglion su Bassani e il fenomeno del "fascismo ebraico" che spiega il suo isolamento nel dopoguerra (Giorgio Bassani, la storia e il paesaggio, pp. 101-112), gli altri articoli trattano di testi in lingua francese che vanno dai primi anni Sessanta al 2016, affrontando la questione della trasmissione dell'eredità ebraica e del trauma in una prospettiva intergenerazionale. A proposito di Jabès, nato nel 1919, e del suo Livre des questions inteso come «entretien infini» (p. 20) con i testi sacri dell'ebraismo, Maxime DECOUT (Edmond Jabès, l'Alphabet contre la Shoah, pp. 15-27) muove da considerazioni sull'assenza come modalità di presenza del divino e sul nome di Dio - tetragramma impronunciabile, assoluto - per mostrare come nella scrittura si manifesti una concezione animata, respiratoria della lettera e della parola che è riaffermazione di singolarità in risposta al «naufrage d'Auschwitz» (p. 26). A partire dalla contrapposizione di Oriente e Occidente ricorrente nei libri di Cohen (1895-1981) che hanno per protagonista Solal, Clara LÉVY mette in rilievo le oscillazioni tra fervore pagano e intransigente ateismo. Da un lato, la rielaborazione degli stereotipi dell'eletto e dell'escluso rivela un'impronta giudaica insieme esibita e combattuta; dall'altro, gli attacchi contro la religione e la cultura ebraiche rasentano l'antisemitismo (Attachement flamboyant et virulence critique: la judéité sans judaïsme d'Albert Cohen, pp. 29-42).

3 Alla generazione designata da Susan Suleiman come 1.5, quella degli enfants cachés, sono dedicati vari studi. È la generazione di Doubrovsky (1928-2017), di Perec (1936-1982) e della cantautrice Barbara (1930-1997), qui accostata a Modiano, nato nel 1945. In merito ai ricordi del pioniere dell'autofiction, scisso tra il «franco-judaïsme assimilateur» (p. 47) della madre e la pienezza giudaica del padre, autentificata dallo yiddish parlato nella sua "tribù", Nelly wolf individua le tracce, sporadiche ma persistenti, di una lingua né viva né morta, al contempo ignota e foneticamente familiare («Pas ma langue»: la présence-absence du yddish dans l'œuvre de Serge Doubrovsky, pp. 43-51). Evidenziando il sofferto divario tra «judéité de filiation» (p. 44) e stigmatizzante «judéité d'assignation» (p. 43) - l'imposizione della stella gialla e la reclusione -, la studiosa traccia la mappa «d'un yiddishland intime, et pourtant interdit» (p. 46). Del secondo, in Perec et la j(e)udéité, premiers écrits (pp. 53-60), vengono investigati gli scritti giovanili L'attentat de Sarajevo e Le condottière. Nella misura in cui vi si profila la poetica della lacuna che emergerà in La disparition o $W$ ou le souvenir d'enfance, in questi testi dell'autore di Ellis Island Francesca DAINESE rinviene «une première esquisse des processus de censure et de résurgence permettant de contourner, de dire autrement l'indicible de la perte» (p. 56). All'immaginario dell'Occupazione che in Modiano, figlio di un ebreo collaborazionista, configura una sorta di «judéité substitutive, à la manière d'un discours d'escorte d'abord expressionniste, ensuite lazaréen», fa da controcanto «l'autofiction lyrique» (p. 62) di Barbara nel suggestivo parallelo proposto da Bruno BLANCKEMAN in La gorge nouée (sur quelques romans de Patrick Modiano et quelques chansons de Barbara) (pp. 61-67): la metafora del nodo in gola veicola l'«irrésolution poétique» (p. 61), tutta reticenza e silenzi, che contraddistingue i testi di entrambi. In Hélène Cixous et l'écriture de la postmémoire ("Gare d'Osnabrück à Jérusalem") (pp. 81-93), Annelies schULTE-NORDHOLT analizza il testo in cui la scrittrice settantottenne rivisita, non la propria infanzia algerina sotto il regime di Vichy, bensì la città tedesca che sua madre, da poco 
scomparsa (Homère est morte) e sua nonna hanno lasciato a causa delle persecuzioni naziste. Scevra da implicazioni religiose, l'appropriazione del passato avviene in modo frammentario, complici oggetti metonimici quali le fotografie o i bicchieri di cristallo per la Notte dei cristalli del Reich. Trasmessa sotto forma di racconto, la memoria personale e storica è già narrazione, letteratura, una letteratura che affonda le sue radici nelle macerie di una città distrutta. Se a Osnabrück si sovrappone Orano, a Troia si sovrappone Gerusalemme, facendo della città in rovina una città promessa.

Alla postmemoria - nozione teorizzata da Marianne Hirsch - eminentemente narrativa della "génération d'après» (A. Schulte Nordholt) s'intreccia lo spinoso dibattito sulla legittimità etica del ricorso, in questo contesto, all'(auto)finzione. È quanto illustra Elena QUAGLIA soffermandosi su Marc Weitzmann lettore di Primo Levi e sulla duplicità della figura di Ulisse (Ulysse, le menteur: la judéité face au négationnisme, de Primo Levi à Marc Weitzmann, pp. 95-100). In Chaos, lo scrittore, classe 1959, contesta e allo stesso tempo sfrutta le risorse dell'autofiction mettendo il narratore sulle tracce del fratello antisemita che firma messaggi negazionisti col nome dell'autore. Ne consegue un'aporetica sovraimpressione identitaria che estremizza le tensioni caratteristiche di una judéité sfuggente e plurale, residuale eppure pervasiva. Di saggio in saggio, tra testimonianza e immaginazione, tra riappropriazione e distanziamento, essa si definisce spesso in negativo, per opposizione: nel caso di Doubrovsky, la «définition par autrui» (p. 47) dettata dall'antisemitismo di Vichy infligge un contenuto a un'identità ebraica volatile, che la scrittura interroga e circoscrive; nei Mémoires interrompus di Barbara si legge: «Je n'avais ni honte ni fierté particulière d'être juive, mais le fait de lire ma singularité dans le regard des autres me rendait agressive» (p. 64). L'identificazione contrastiva diventa motore di azione teatrale in Pour en finir avec la question juive, parabola comica su cui si concentra Jean-Paul DUFIET in Le discours de la judéité chez Jean-Claude Grumberg (pp. 69-80). Attingendo ai modelli del dialogo filosofico e della pièce à idées, il drammaturgo, nato del 1939 e figlio ateo di una vittima della Shoah, mette a confronto due vicini: l'uno ebreo nativo e non credente, l'altro cattolico e prevenuto contro gli ebrei, ma che alla religione ebraica finisce per convertirsi. Sulla scena della judéité e della judéophobie, l'ostilità si fa interdipendenza: «Le Juif athée assimilé défend le Juif traditionnaliste qu'il n'est pas, parce que celui-ci lui donne la possibilité d'être juif tout en étant athée et assimilé» (p. 80). Emerge, in definitiva, l'impossibilità - e l'insensatezza - di attribuire all'identità ebraica significati stabili e univoci. 